\title{
Il sacro degli altri. Culti e pratiche rituali dei migranti in Sicilia
}

Edizioni Museo Pasqualino, Palermo, 2018

\section{Gabriella D'Agostino}

\section{OpenEdition}

\section{Journals}

Edizione digitale

URL: http://journals.openedition.org/aam/2340

DOI: $10.4000 /$ aam. 2340

ISSN: 2038-3215

\section{Editore}

Dipartimento Culture e Società - Università di Palermo

Notizia bibliografica digitale

Gabriella D'Agostino, « II sacro degli altri. Culti e pratiche rituali dei migranti in Sicilia », Archivio antropologico mediterraneo [Online], Anno XXII, n. 21 (2) | 2019, online dal 31 décembre 2019, consultato il 23 septembre 2020. URL : http://journals.openedition.org/aam/2340 ; DOI : https:// doi.org/10.4000/aam.2340

Questo documento è stato generato automaticamente il 23 settembre 2020.

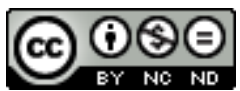

Archivio antropologico mediterraneo è distribuita con Licenza Creative Commons Attribuzione - Non commerciale - Non opere derivate 4.0 Internazionale. 


\section{Il sacro degli altri. Culti e pratiche rituali dei migranti in Sicilia}

Edizioni Museo Pasqualino, Palermo, 2018

Gabriella D'Agostino

NOTIZIA

Il sacro degli altri. Culti e pratiche rituali dei migranti in Sicilia, Edizioni Museo Pasqualino, Palermo, 2018.

ISBN 978-8897035398 
1 Nel novembre del 2017 la Fondazione Buttitta di Palermo inaugurava la mostra "Il sacro degli altri. Culti e pratiche dei migranti in Sicilia", riproposta nei mesi successivi in sedi diverse. Nel luglio del 2018 la mostra venne allestita nell'ambito della XIII edizione del Festival internazionale di documentari Sole Luna Doc Film Festival e fu realizzato il catalogo. L'ultimo allestimento è stato presentato in occasione del Convegno "Migrare", organizzato dall'Università degli Studi di Palermo nel maggio 2019.

2 Mostra e catalogo rappresentano un osservatorio privilegiato sulla dimensione interreligiosa nei contesti urbani delle nostre società contemporanee grazie alle fotografie scattate su tutto il territorio

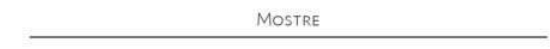

II SACHBO DBGI ALTIRI

Culti e pratiche rituali dei migranti in Sicilia

fotografie di Attilio Russo e Giuseppe Muccio

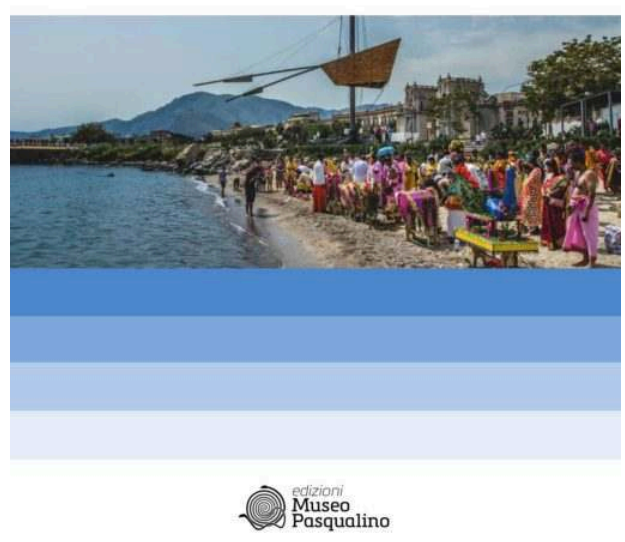
siciliano da due fotografi professionisti, Attilio Russo, messinese, e Giuseppe Muccio, siracusano. I fotografi hanno documentato a tutto campo, per decenni, i culti e le pratiche festive "tradizionali" della nostra regione costituendo un archivio di migliaia di foto di estremo interesse. Precocemente rispetto a un fenomeno che avrebbe assunto la dimensione mediatica che oggi conosciamo, Russo e Muccio nel corso delle loro campagne di documentazione hanno cominciato a cogliere quanto significativa fosse la presenza di pratiche religiose "altre" e testimoniato visivamente il fenomeno sin dal suo primo manifestarsi nelle città siciliane. Con discrezione, hanno infatti cominciato a fotografare culti e pratiche delle comunità di migranti quando esse erano praticate in modo pressoché esclusivo dagli attori sociali delle comunità interessate, fuori dai percorsi turistici che avrebbero caratterizzato almeno alcune di queste manifestazioni qualche anno dopo.

Il catalogo propone dunque, a distanza ravvicinata, una panoramica sui culti che fanno parte del nostro orizzonte contemporaneo, nei nostri territori, e ha l'obiettivo di costringerci a una torsione che ci conduca a trasformare noi negli altri e gli altri in noi, non per una infruttuosa e inconsistente retorica dell'assimilazione e della cosiddetta integrazione, ma per mostrare appunto come la distanza tra noi e gli altri sia innanzitutto mentale. La selezione delle immagini è stata guidata proprio da questa esigenza: declinare il sacro degli altri, ritagliarne gli spazi, per aree di contiguità e prossimità sino a includere ciò che sembra configurarsi come il più radicalmente altro. Le immagini riguardano infatti culti di cristiani ortodossi, ebrei, musulmani, induisti, buddisti, a Palermo, Catania, Siracusa. Il punto di osservazione è un 'noi' che condivide, per storia e territorio, una certa tradizione religiosa, quella cattolica, ma questo orizzonte in mostra resta sottinteso, è assente, perché è 'materia' del nostro immaginario e della nostra memoria, filtro, consapevole o inconsapevole, attraverso cui osserviamo e percepiamo la realtà e il presente. Nel catalogo questa 'omissione visiva' è colmata dal saggio di Ignazio Buttitta che sintetizza un'attività di ricerca sul campo che, come è noto, dura da più di un ventennio («Cosa fa la differenza? Uno sguardo sul "nostro" sacro»). Si tratta di una chiave di lettura consolidata, incentrata sulla 
dimensione del tempo sacro e sulla costruzione dell'appaesamento', del radicamento nello spazio, attraverso l'individuazione di forme stratificate e rintracciabili nel corso del tempo rispetto a cui l'Autore individua una fenomenologia, per chiedersi, per esempio, in che modo sia possibile mettere in relazione lo statuto del divino della tradizione induista e del cattolicesimo "popolare". Osserva Buttitta: «Le figurazioni divine della tradizione induista [...] - contrariamente a quanto avviene per i cristi, i santi e le madonne la cui santità e appartenenza alla sfera del sacro è, quando lo è, per lo più evidenziata dall'aureola e da un corredo simbolico affatto "naturale" - sono dotate di caratteristiche iconiche che ne denunziano subitaneamente, almeno all'occhio occidentale, la radicale "alterità", la assoluta distanza dall'umano, il rifiuto del mistero dell'incarnazione: figure "smisurate", mostruose se non grottesche, i volti e le membra molteplici, la stessa pigmentazione dell'epidermide, caratteri ferini, corredo simbolico inusuale e fantastico». Tuttavia, si tratta davvero di dimensioni del sacro "incommensurabili"? «Non vi sono luoghi, momenti, situazioni - si interroga Buttitta in cui questa [distanza] può ridursi addirittura facendoci apparire familiari i gesti, le forme, i suoni, le parole degli altri? Nonostante il loro "singolare" apparire - continua Buttitta - le divinità induiste, almeno nel sentire comune dei credenti, non sono estranee all'immanenza, sono "umanamente" vicine ai loro devoti. Al di là di ogni speculazione e rappresentazione filosofica, esse infatti agiscono e sentono tra gli uomini e come gli uomini. Intervengono cioè nelle loro vite e ne condizionano gli eventi, non diversamente dai santi del "pantheon" del cristianesimo popolare» (p. 15).

La riflessione che Buttitta propone mette in luce, da un lato, esempi di continuità temporale in territori siciliani interessati nell'antichità, per esempio, da culti a divinità femminili che, nel corso dei secoli, hanno mantenuto alcuni tratti formali seppur rifunzionalizzati. Dall'altro, azioni quali la dimensione agonale, le prove di forza e di coraggio, le danze e le corse, gli interventi di forze ctonie, il mascheramento, le diverse forme di mortificazione del corpo e gli atti penitenziali, l'offerta e la condivisione del cibo, ma anche le pratiche dell'astinenza scandiscono trasversalmente la fenomenologia delle azioni festive, rendendo labile quel confine tra il nostro sacro e quello degli altri. «Non si tratta dunque - avverte Buttitta - di cercare analogie e derivazioni tra diversi istituti festivi, prassi cultuali, credenze o figure divine, né di tentare di ricostruire qualche forma arcaica di religione universalmente diffusa; semmai, di sottolineare un'analoga religiosità nel senso di una condivisione di vocaboli, di simboli rituali derivata dalle comuni istanze connesse ai bisogni di protezione, riproduzione, nutrizione, da una comune attenzione alle trasformazioni della realtà in quanto regolata da entità trascendenti con le quali è necessario intessere un positivo rapporto di reciprocità» (p. 22).

5 A un livello ancora più profondo, strutturale, si interroga Salvatore D'Onofrio nel suo contributo, «La lezione dei germogli hopi», che propone una sintesi dell'ultimo capitolo di un suo recente lavoro, incentrato sullo studio dell'uso rituale dei germogli di grano, orzo, legumi o altri semi, fatti germogliare in luoghi chiusi, che caratterizzano un orizzonte spazio-temporale molto ampio. D'Onofrio, partendo dall'ipotesi che queste pratiche abbiano una connessione originaria con il culto zoroastriano, prende in esame la loro diffusione dall'India all'Europa, sia in riferimento al passato sia al presente, e ne individua le invarianti simboliche (S. D'Onofrio, Le matin des dieux: Du Norouz persan aux Pâques chrétiennes, Mimésis, Paris 2018). Da Teheran a Parigi (XV arrondissement) per la festa di Norouz, il capo d'anno persiano, alla Pasqua di Nocera Terinese in Calabria e in molte altre città del meridione italiano e in Sicilia, grano, orzo, miglio vengono fatti 
germinare, alla luce o al buio, dalle donne, nel momento critico del passaggio da un tempo consumato a un tempo rigenerato, affermando la vita sulla morte. «Da qui avverte D'Onofrio - l'esigenza di cogliere le costanti e le variabili [...]: per esempio, la rinascita della natura e del tempo non sempre si inquadra nel mitologema di morte e resurrezione di un dio salvatore [...] ma si dispiega nell'ambito di sistemi i cui segni, in tutto o in parte, sono suscettibili di combinazioni le più diverse» (S. D'Onofrio, op. cit. p. 14). In questo orizzonte, D'Onofrio si interroga «sul quadro rituale che conferisce ai germogli la loro efficacia simbolica e sull'orizzonte mitico che li giustifica ideologicamente» (D'Onofrio, op. cit. p. 20). Con l'esempio "decentrato" dei germogli hopi, D'Onofrio conclude un percorso che ha attraversato il mondo, temporalmente e spazialmente: «dagli Hindu agli Hopi, passando per gli Zoroastriani o i Greci, i Provenzali, i Sardi [...] [attraverso] la lunga durata dei riti e dei miti che sono loro soggiacenti, i germogli resistono, le barriere religiose e i venti di guerra - passati e presenti - non sono riusciti a sradicarli dalle culture che li accolgono. [...] è la forza simbolica dei processi vitali e degli esseri naturali che si impone: la memoria della terra, il ritorno delle stagioni, il concatenarsi delle generazioni» (p. 36).

6 I contributi cui abbiamo fatto riferimento colgono bene fenomenologia e morfologia del sacro e le logiche combinatorie del pensiero mitico e rituale. Questi saggi sono la premessa e l'introduzione al percorso fotografico proposto mentre i saggi che seguono ne costituiscono la parte integrante a corredo delle immagini. Così, il saggio di Maria Rizzuto, "Venerdì Santo ortodosso», si incentra sul momento più significativo del calendario liturgico ortodosso, la Settimana Santa che conduce alla Pasqua, nel mondo bizantino denominata "Grande e Santa Settimana". Le foto in mostra riguardano in particolare il Venerdì Santo celebrato nella Chiesa ortodossa costantinopolitana di San Leone a Catania. L'autrice indaga la centralità della simbologia della luce e descrive l'articolazione dei tre principali Uffici che si svolgono il venerdì santo: il "Mattutino delle sante sofferenze del nostro Signore Gesù Cristo"; l"“Ufficio delle Grandi Ore", o "Ufficio delle Ore Regali" cui segue direttamente il "Vespro della Deposizione"; il "Mattutino della Sepoltura".

7 Luciana Pepi è l'autrice del contributo «Purim», la festa del "capovolgimento delle sorti". Pepi rievoca le vicende che sono all'origine della sua istituzione (Ester, 9,20-26), lo scampato sterminio degli ebrei da parte di Aman, consigliere del re di Persia Assuero, grazie all'astuzia di Mardocheo e Ester. Celebrata sia in sinagoga sia nelle case private il 14 di Adar (nome del mese secondo il calendario ebraico corrispondente a febbraiomarzo), è «una festa modana, una festa di gioia, di allegria e si celebra con canti, balli, banchetti. In questo giorno grande importanza riveste la solidarietà sociale: per poter essere veramente contenti bisogna far felici [...] gli altri, specialmente quando non sono abbienti. Per questo si mandano regali agli amici e si fanno doni ai poveri. [...] Un posto fondamentale ha il banchetto festivo [...]. La tavola deve essere imbandita e ornata con candele accese, perché la gioia deve essere accompagnata dalla luce. Durante il pasto si cantano inni [e bisogna] bere in abbondanza» (pp. 48-49). Il catalogo documenta alcuni momenti di questa festività celebrata a Siracusa, con immagini della lettura del libro di Ester dal rotolo di pergamena manoscritto, la Meghillà Ester.

8 Patrizia Spallino con il saggio «Ramadān» e Federico Salvaggio con «Il digiuno di Ramadān. Tra unità religiosa e diversità culturale. Una testimonianza "eccentrica"” introducono alla festa della fine del mese di digiuno (il nono mese lunare dell'égira), osservata in tutto il mondo musulmano. Chiamato da Maometto "il mese della mia 
comunità", la sua fine è salutata al grido di Ramadān karīm! Ramadān mubārak! (R. generoso! R. benedetto!). Nei paesi musulmani il mese del Ramadān si caratterizza per un'atmosfera speciale: «Qualche ora prima del tramonto [...] un silenzio irreale scende sulle città: commercianti e artigiani chiudono le saracinesche dei negozi e laboratori, gli ultimi tassisti accelerano per le vie correndo all'impazzata per rientrare alle proprie abitazioni, nessun mezzo di trasporto circola, uffici e scuole sono già chiusi da ore, palestre e centri di ritrovo deserti, mentre da finestre e balconi spalancati risuonano voci domestiche e tintinnii di stoviglie. [...] Al colpo di cannone ancora silenzio, il silenzio creato dalle bocche che mangiano e bevono, seguito da sospiri di sollievo e soddisfazione [...] dopo [...] tutta l'atmosfera riprende vita e la gente si riversa per strade e piazze illuminate tornando a transitare in vetture strombazzanti fino a formare per la gioia traffico e ingorghi (p. 57).

9 Il tempo del Ramadān fuori dai contesti musulmani non può passare inosservato anche nei luoghi in cui la presenza musulmana, pur numericamente consistente, sia pur sempre marginale. Nelle città siciliane, e in particolare a Palermo e a Catania, anche un osservatore distratto noterà infatti almeno la fine del periodo di digiuno, quando $\mathrm{i}$ fedeli, curati nell'aspetto e negli abiti, si recheranno in moschea, a Catania, e alla spianata del Foro Italico, a Palermo, sul lungomare, per pregare. Le immagini in catalogo documentano questi momenti.

10 Le celebrazioni di Katina Pinkama e Vesak sono il tema del contributo di Emilio Ajovalasit e sono illustrate nelle foto che riguardano questi culti della tradizione buddista in Sicilia, con particolare riferimento a Catania e a Comiso. La prima appartiene alla tradizione theravada ed è una festa che «rinsalda il legame tra la comunità laica e quella dei monaci» (p. 71). Buddha Gautama stabilì che i monaci dovevano alternare ai viaggi per la diffusione dell'insegnamento dei periodi stanziali dedicati alla meditazione e al sostegno spirituale delle comunità del territorio. Katina Pinkana è il giorno che pone fine al periodo di ritiro. Vesak è la festa che celebra la nascita, l'illuminazione e la morte di Buddha. Nonostante le diverse tradizioni che interessano il pensiero buddhista, questa festa accomuna la gran parte di esse. In questa occasione, la comunità laica fa visita ai monaci recando loro offerte e assistendo alla recitazione dei sutra, spesso accompagnata da strumenti musicali a percussione o di altro tipo. Il canto dei sutra è un aspetto fondamentale della pratica buddhista: «Secondo le varie tradizioni [...] la recitazione delle parole del Buddha o di altri Maestri ha ripercussioni positive sull'intero universo contribuendo ad alleviare la sofferenza degli esseri sensibili e a diffondere pace e benessere» (p. 73). A Comiso la Pagoda della Pace dove viene celebrato Vesak è stata fondata dal monaco Morishita in seguito alle manifestazioni contro la vicina base NATO, a favore della pace e della non violenza. La Pagoda è un grande stupa, l'unica di questo genere sul territorio italiano e tra le poche in Europa. Qui ogni giorno il monaco Morishita prega e apre il tempio a chiunque voglia partecipare.

11 Il catalogo infine propone una selezione di immagini di celebrazioni rituali della comunità indù mauriziana e tamil, la cui articolata composizione è ben descritta nel saggio di Giulia Viani («"Induismi” di Sicilia. I riti mauriziani e srilankesi in onore di Shiva, Ganesh, Murigan»). Le comunità più numerose sul territorio siciliano sono quella mauriziana, suddivisa in hindu, marathi, telegu e tamil, e srilankese (tamil) e sono giunte in Sicilia circa 25 anni fa con una concentrazione prevalente a Catania e a Palermo. In entrambe le città non ci sono templi veri e propri ma luoghi adattati al 
culto di cui si prendono cura le diverse associazioni culturali in cui sono riunite le persone delle comunità interessate. Statue, tappeti, suppellettili e arredi sacri provengono dall'India o dai luoghi di origine dei migranti che ricreano il contesto adatto alla celebrazione del loro calendario festivo e rituale articolato e complesso. Le foto documentano il rito di Shivaratri celebrato dagli hindu mauriziani di Catania, la processione di Ganesha Chaturthi agita dai marathi mauriziani di Palermo e Catania, i riti tamil del Cavedee dei mauriziani e degli srilankesi che vivono a Palermo.

Shivaratri è la "notte di Shiva". Dio distruttore dell'universo, egli è anche il dio della rigenerazione e della rinascita. Il linga è il suo simbolo, posto al centro di ogni tempio induista. Con cadenza mensile la pietra nera a forma di fallo è oggetto di culto da parte della comunità, mentre la "Grande notte", la festa di Maha Shivaratri ha cadenza annuale e cade tra febbraio e marzo tra il tredicesimo e il quattordicesimo giorno della luna nera di phaalguna. I fedeli si preparano per nove giorni assumendo cibo vegetariano e durante la notte offrono al linga fiori, frutta, cibo sacro (prasad), la benedizione con il fuoco. Il linga viene purificato con acqua, latte, miele, burro fuso chiarificato (ghee) e adornato con ghirlande di fiori bianchi. A portare le offerte è una coppia guidata dall'officiante (pandit). Poi, il resto degli astanti può portare la propria offerta, una noce di cocco che ognuno rompe sul linga, mentre si intonano canti. Il linga viene ricoperto con una stoffa bianca fissata con un laccio intinto nello zafferano, segnato da una polvere grigia che rappresenta la cenere dei morti (vibuthi) e adornato con il malah, la coroncina di legno di bambù. Si veglia tutta la notte sino all'alba.

Ganesh è il dio «invocato dai suoi devoti come colui che aiuta a oltrepassare tutti gli ostacoli e [...] a scopo propiziatorio prima di iniziare qualsiasi attività» (p. 82). La festa a lui dedicata è molto importante e partecipata e culmina con una processione verso il mare a cui si affideranno statue di gesso e terracotta raffiguranti il dio. A Catania la destinazione finale è la Playa, la spiaggia della città, a Palermo è quella della borgata marinara dell'Arenella-Vergine Maria. La sacralizzazione dello spazio esterno passa attraverso un percorso in ginocchio da parte dei devoti, che recano le pesanti statue di Ganesh sulle loro teste, da soste agli incroci presso cui si accendono piccoli fuochi e si rompono noci di cocco i cui pezzi vengono consumati sul posto o portati a casa, da un corteo di suonatori e danzatori, da canti intonati dai più giovani che danzano saltando accompagnati dal battere ritmato delle mani delle donne che si dispongono intorno a loro in cerchio. Giunti sulla spiaggia, si allestisce un altare mentre i devoti si purificano in acqua e purificano il mare lanciando una noce di cocco che brucia, fiori e frutta. Coloro che conducono le statue entrano in acqua dando le spalle all'orizzonte mentre gli astanti invocano Ganapati (Ganesh nella funzione di "capo delle schiere celesti”) e il ritorno dell'anno successivo.

14 I riti del Cavadee riguardano la capacità di sopportare il dolore. Il termine significa "fardello" e nei riti si tratta di pesanti strutture di legno di bambù portate dai devoti in processione per un voto fatto in precedenza riguardante l'intervento del dio per motivi di salute, il successo del proprio figlio, ogni momento difficile della propria esistenza in cui le forze personali non sono sufficienti a raggiungere lo scopo desiderato. Al trasporto del cavadee si associano anche interventi sul corpo, in particolare sulla lingua e sulle guance, conficcando aghi d'argento «a forma di miniature di vel, simbolo del potere di Murugan» a cui può accompagnarsi anche l'imbavagliamento, a testimoniare la rinuncia alla facoltà di parola da parte dei penitenti. Al cavadee «possono associarsi riti similari, come camminare sulle braci ardenti e su scale di coltelli [...]. Prima di 
compiere il rito, il penitente deve sottoporsi a un lungo periodo di purificazione per preparare la mente e il corpo ad accostarsi al divino [...]. Il digiuno prevede una dieta strettamente vegetariana, il dormire per terra, il divieto di indossare scarpe e indumenti di pelle, l'astinenza sessuale e l'obbligo di non toccare nessuno per mantenere la purezza necessaria al rituale; anche gli eventuali consorti devono seguire le stesse pratiche» (p. 85). Il rito si compie dopo undici giorni di digiuno e di preghiera e culmina con un rito che si svolge sul lungomare dove i cavadee vengono depositati, allineati e consacrati dal pandit. Qui l'officiante interviene con gli aghi su lingua e guance, sottraendo il devoto alla vista degli astanti mentre compie la perforazione rituale sotto un lenzuolo bianco. Il penitente viene incitato da grida sempre più forti $\mathrm{e}$ ripetute ("aro-hara") sino a indurgli uno stato di trance che si manifesta con l'abbandono a danze estatiche. È questo il momento in cui i cavadee, pesanti e adorni di fiori, frutta, piume di pavone (l'uccello-cavalcatura di Murugan) vengono posti sulle spalle dei penitenti che lasciano la spiaggia e si avviano a percorrere le strade cittadine per fare ritorno al tempio, ballando e dimenandosi per lo stato di trance indotto. Nel tempio, che si raggiunge dopo circa un paio di ore, il cavadee viene tolto dalle spalle del devoto, le offerte vengono donate al dio e il penitente ha adempiuto al proprio voto. $\mathrm{Si}$ possono adesso togliere gli aghi rituali e sui fori si spalma cenere sacra. Se queste sono le fasi principali del rito così come praticato dai mauriziani, quelle del rito celebrato dagli srilankesi presentano momenti in cui la mortificazione corporale è maggiore. Le parti del corpo su cui si interviene con gli aghi e anche con uncini, qui spesso di maggiori dimensioni, riguardano anche la lingua, la fronte, la schiena. Come commenta Viani: «I sacrifici del corpo previsti durante il rito costituiscono modalità privilegiate per raggiungere stadi ulteriori di conoscenza. L'uomo si sperimenta nella sua corporeità e in tutte le culture il corpo è un elemento fondamentale, luogo di contatto $\mathrm{e}$ di separazione tra sé e il mondo: siamo di fronte al linguaggio arcaico che pone il corpo al centro, il dono e il sacrificio della propria energia fisica al dio con cui ci si mette in comunicazione. L'automortificazione traduce l'esigenza di trascendere i limiti della condizione umana e la trance agisce come mezzo per esplorarli, sperimentando e superando la sofferenza». Il dolore, continua Viani, «gioca un ruolo fondamentale in tutte le religioni; esse non lo anestetizzano, ma insegnano "l'arte del soffrire" e la disciplina del corpo per raggiungere una maggiore consapevolezza spirituale. I penitenti dimostrano a se stessi e agli altri la propria capacità di lottare e sopportare il male fisico, di superare la corporeità stessa e la sua materialità» (p. 87).

Questa sintesi mostra evidenti punti di intersezione e simbologie ricorrenti in rituali in prima battuta percepiti come distanti e diversi le cui grammatica e sintassi bisogna imparare a riconoscere. In questa direzione, sarebbe opportuno recuperare la lezione di Raimundo Panikkar su quanto egli ha chiamato "equivalenti omeomorfici", intendendo con questa espressione il modo in cui certi principi, considerati basilari per una tradizione culturale, possono trovare un "equivalente funzionale" in un'altra tradizione. Non si tratta tanto di individuare analogie tra principi quanto piuttosto di ricercare gli spazi omologhi da essi occupati nei rispettivi sistemi di riferimento, omologhe correlazioni di funzione. Forme simili, dunque, tra specifiche credenze di tradizioni religiose diverse, secondo un metodo non più "imperativo" ma "comparativo". Pannikar ha infatti espresso una severa critica in relazione a quelle filosofie e teologie che hanno assunto la propria posizione come il punto di vista obiettivo e confessionale per eccellenza a partire dal quale guardare a ogni altro sistema di pensiero, definendo questo approccio "colonialismo intellettuale", 
espressione della "sindrome occidentale" assetata di universalità. L'equivalenza omeomorfica, di contro, può essere uno strumento concettuale fruttuoso perché mentre lascia cogliere la specificità di una forma religiosa, consente di cercare correlazioni andando oltre la semplice analogia.

Se il catalogo della mostra è una sintesi interessante di traiettorie di ricerca fin qui esplorate, restano altre piste che devono essere indagate fruttuosamente anche ai fini di un dialogo interreligioso realmente finalizzato alla comprensione reciproca. Esso riguarda il modo in cui gli scenari rituali possono produrre forme di socialità. A partire da qui possono aprirsi nuove prospettive per leggere le modalità di interazione tra pratiche rituali di determinate comunità di migranti e le altre comunità che compongono il panorama sociale. Il caso di Palermo può essere assunto come esempio spingendo a un'indagine sugli spazi-tempi delle pratiche religiose (culti collettivi, devozioni personali) con cui alcune comunità migranti si 'intrecciano' nelle maglie dello spazio urbano della città di Palermo e nelle sue diverse temporalità. Lavoro, feste collettive, tempi liberi: "i culti" si stabiliscono come ritmi che ad un tempo ancorano agli spazi della città, li rivelano diversamente ma anche si integrano, eccedendoli, ai diversi suoi calendari. In questo modo le pratiche religiose così spazio-temporalmente situate possono consentire anche di analizzare il modo in cui esse diventano il medium per azioni di networking sia rispetto ai luoghi di residenza sia rispetto ai luoghi di origine e/o provenienza. Per esempio, riguardo alle "comunità" srilankese e mauriziana di religione indù, c'è una condivisione degli spazi di culto (riguardo alla gestione economica e alla loro manutenzione), anche se le cerimonie vengono celebrate separatamente e nella composizione dei gruppi di devoti prevale la formazione su base "etnica". Entrambe le comunità tuttavia sono presenti nello spazio pubblico del pellegrinaggio al santuario di Santa Rosalia lungo Monte Pellegrino, sia il 4 settembre, data canonica del pellegrinaggio (acchianata, salita), sia nella ascesa al Monte che i singoli fedeli fanno la domenica mattina, 'mescolati' ai palermitani "autoctoni". A questa pratica devozionale partecipano anche i tamil di religione cattolica che, invece, per la gestione ordinaria del culto, si sono ritagliati uno spazio di autonomia rispetto ad altri cattolici. Essi infatti hanno una propria chiesa di riferimento e un officiante in lingua tamil.

Individuare dove i culti hanno luogo nella città, può dire qualcosa sul modo di risiedere nella città, ossia sul modo d'interagire con il contesto sociale e simbolico di residenza, consentendo di cogliere i ritmi del culto, cosa "dicono" e come si inseriscono nelle scansioni della vita ordinaria dei migranti nella città. Significativo può risultare, inoltre, interrogarsi sulle 'forme' della credenza, indagare possibili trasformazioni del rito e del culto quale effetto e matrice dei legami intessuti con il territorio di riferimento. Ricostruire i calendari festivi, le modalità di interazione dei fedeli nei luoghi di culto, nell'alternanza o compresenza di spazi pubblici e privati, nelle dinamiche di prossimità o distanza rispetto a cerimonie religiose radicate nel territorio (per es., la partecipazione al Festino, la festa di santa Rosalia nell'occorrenza del 14 luglio e 15 luglio) può aprire scenari ulteriori accanto a quelli già esplorati che certamente condurrebbero a una maggiore prossimità conoscitiva, intellettuale ed emozionale del "sacro degli altri".

18 In altri termini, riflettere sulla modalità di produzione di spazi di accettazione/ inclusione, o anche di esclusione, di cui i processi rituali sono capaci, può portare a interrogarsi sul ruolo che la religiosità e le pratiche devozionali giocano non solo sul 
processo di appaesamento di gruppi identificabili per omogeneità "culturale" o "etnica", ma anche sulla dimensione della permeabilità dei contesti in cui queste pratiche prendono forma, su eventuali situazioni di subalternità sociale, sulla percezione pubblica della loro presenza. Non è raro che nella costruzione del processo di appaesamento attraverso la partecipazione a pratiche cerimoniali in situazione migratoria, si producano dinamiche di costruzione della visibilità pubblica non solo rispetto al territorio di approdo ma anche a quello di provenienza. La possibilità di essere qui e altrove offerta dalle tecnologie informatiche delinea scenari in cui la "doppia assenza" di sayadiana memoria si configura sempre più spesso come "doppia presenza" aprendo un'ulteriore prospettiva di riflessione sui modi inediti dell'esserci, di abitare, di costruirsi come individui e come collettivi, di performare appartenenze e credenze civico-religiose.

L'appartenenza religiosa, dimensione del vivere talvolta maggiormente enfatizzata più che nei territori di origine perché funzionale alle strategie di appaesamento, può essere percepita come tratto identitario ascritto o acquisito a seconda delle situazioni della vita ordinaria in relazione alle diverse istituzioni attraverso cui il migrante transita, scuola, luogo di lavoro, ospedali, istituzioni cittadine. La dimensione della "religione vissuta" può dunque essere considerata una variabile importante per comprendere il tipo di partecipazione e di coinvolgimento rispetto alla vita, alla politica e alla poetica dei luoghi di residenza. Un osservatorio interessante, da questo punto di vista, è rappresentato dalla Consulta delle Culture della città di Palermo, un organismo consultivo e propositivo dell'amministrazione comunale, nato nel 2013, che rappresenta tutti gli stranieri residenti a Palermo, comunitari ed extracomunitari, composto da 21 membri eletti che eleggono un presidente. Potrebbe rivelarsi interessante studiare etnograficamente il processo di selezione della rappresentanza all'interno della comunità di riferimento, anche in una prospettiva di genere, il tipo di supporto fornito dai diversi gruppi rispetto ai propri candidati durante la campagna elettorale, in che modo i momenti cerimoniali e festivi della comunità di riferimento abbiano un ruolo nella costruzione della visibilità pubblica dei candidati. In altri termini, se e come la condivisione religiosa agisca come risorsa per la mobilitazione politica e come, nella vita quotidiana, essa si traduca in atti concreti di solidarietà, supporto, costruzione di una cittadinanza attiva, per quanto non formalmente conseguita. Si tratta, insomma, di individuare piste di ricerca che possano soddisfare l'esigenza conoscitiva degli studiosi e, al tempo stesso, fornire un solido supporto a quanti sono impegnati a vario titolo e nei diversi ambiti a costruire una società aperta $\mathrm{e}$ plurale in cui non ci sia posto per retoriche e politiche vili e pericolose per il futuro dell'intera umanità. 